\title{
Gelatin-chondroitin-hyaluronan tri-copolymer scaffold for cartilage tissue engineering
}

\author{
Chih-Hung Chang ${ }^{\mathrm{a}, \mathrm{b}}$, Hwa-Chang Liu ${ }^{\mathrm{a}, \mathrm{c}}$, Chien-Cheng Lin ${ }^{\mathrm{a}}$, Cheng-Hung Chou ${ }^{\mathrm{a}}$, \\ Feng-Huei $\operatorname{Lin}^{\mathrm{a}, *}$ \\ a Institute of Biomedical Engineering, National Taiwan University, 7 Chung-Shan South Road, Taipei 100, Taiwan, ROC \\ ${ }^{\mathrm{b}}$ Division of Orthopedics, Department of Surgery, Far Eastern Memorial Hospital, Pan-Chiao 220, Taipei, Taiwan, ROC \\ ${ }^{\mathrm{c}}$ Department of Orthopedic Surgery, National Taiwan University Hospital, Taipei 100, Taiwan, ROC
}

Received 26 September 2002; accepted 13 May 2003

\begin{abstract}
The mechanism by which the cell synthesizes and secretes extracellular matrix (ECM) and is, in turn, regulated by the ECM is termed dynamic reciprocity. The aim of the present work was to produce a gelatin/chondoitin-6-sulfate/hyaluronan tri-copolymer to mimic natural cartilage matrix for use as a scaffold for cartilage tissue engineering. The scaffold produced had a uniform pore size of about $180 \mu \mathrm{m}$ and adequate porosity of $75 \%$. Porcine chondrocytes were seeded onto the tri-copolymer scaffold and cultured in Petri dishes or spinner flasks for 2, 3, 4, or 5 weeks. Chondrocytes were uniformly distributed in the scaffold in the spinner flask cultures, but less so in the Petri dish cultures. Secretion of ECM was found under histology examination. In spinner flask cultures, chondrocytes retained their phenotype for at least 5 weeks, as shown immunohistochemically, and synthesized type II collagen. These results show that gelatin/chondroitin sulfate/hyaluronan tri-copolymer has potential for use as a cartilage tissue engineering scaffold.
\end{abstract}

(C) 2003 Elsevier Ltd. All rights reserved.

Keywords: Gelatin; Chondroitin sulfate; Hyaluronan; Cartilage; Tissue engineering

\section{Introduction}

Although articular cartilage is a metabolically active tissue, the chondrocytes in the matrix have a relatively slow state of turnover. The tissue itself lacks a blood supply to support repair and remodeling. Because of the limited capacity for spontaneous repair, minor injury to articular cartilage may lead to progressive damage and degeneration. In older patients with diffuse articular cartilage injury, total joint arthroplasty is the treatment of choice. In young patients with focal articular cartilage injury, autogenous chondrocyte implantation, mosaicplasty, or marrow-stimulating techniques, such as microfracture or multiple drilling, are better alternatives than joint replacement therapy, but the results are variable and the techniques have some limitations [1]. Autogenous chondrocyte implantation with a periosteal

*Corresponding author. Tel.: +886-2-23912641; fax: +886-223940049.

E-mail address: double@ha.mc.ntu.edu.tw (F.-H. Lin). graft has shown encouraging results, but the predictability and reliability of hyaline or fibrocartilage formation are still questionable [2]. Marrow-stimulating techniques result in fibrocartilage formation, which has a lower mechanical strength than hyaline cartilage and only limited repair capacity [3]. For mosaicplasty, the major problems are limited availability of autogenous tissue and donor site morbidity; this procedure also involves destroying healthy non-weight-bearing tissue to treat diseased tissue, and both the donor site and treated area would be expected to degenerate [4].

Recently, tissue engineering has emerged as a new method involving the combining of cells, scaffold, and bioactive agents to fabricate functional new tissue to replace damaged tissue [5]. The advantages of incorporating scaffold into this approach are that the scaffold matrix can provide the initial structural support and retain cells in the defective area and is then degraded when the cells secrete their own matrix, and scaffold matrix can act as a delivery system for bioactive agents, such as growth factors. Many kinds of scaffold, both 
natural and synthetic, have been proposed for use in cartilage tissue engineering [6]. Scaffold plays an important role as the extracellular matrix (ECM) during engineered tissue development, and scaffold with an informational function, e.g., material containing the Arg-Gly-Asp sequence which facilitates cell attachment, should be better than non-informational synthetic polymers $[7,8]$.

The mechanism by which the cell synthesizes and secretes ECM and is then, in turn, regulated by the ECM is termed dynamic reciprocity [9]. Inclusion of chondroitin sulfate in scaffold may promote the secretion of proteoglycan and type II collagen, but may also inhibit mitosis [10]. Hyaluronan is present in high amounts in the ECM during embryonic cartilage development, and also facilitates the integration of engineered cartilage [5]. Since gelatin is basically denatured collagen and presumably retains informational signals, such as the Arg-Gly-Asp sequence, we hypothesized that a tri-copolymer formed from gelatin, chondroitin, and hyaluronan might mimic cartilage matrix and provide the necessary information for cell attachment to meet the requirement for dynamic reciprocity for cartilage tissue engineering.

In this study, a tri-copolymer scaffold was produced from chondroitin-6-sulfate, hyaluronan, and gelatin to mimic cartilage matrix. Preliminary results of in vitro chondrocyte culture in this $3 \mathrm{D}$ scaffold are also described.

\section{Materials and methods}

\subsection{Fabrication of scaffold}

\subsubsection{Preparation of tri-copolymer scaffold}

The percentage dry weight of each component of hyaline cartilage is $15-20 \%$ type II collagen, $5-10 \%$ chondroitin sulfate, and $0.05-0.25 \%$ hyaluronan [11]. We used these percentages to try to make a scaffoldmimicking natural cartilage matrix from gelatin (a denatured collagen), chondoitin-6-sulfate, and hyaluronan, although the percentage of gelatin was slightly reduced to increase scaffold pore size. Gelatin powder (0.5 g, G-2500; Sigma Co., St. Louis, USA), sodium hyaluronate (HA) $(5 \mathrm{mg}, 0.5 \mathrm{ml}$; Seikagaku Co., Tokyo, Japan), and chondroitin-6-sulfate (C6S) powder (0.1 g; Sigma Co., St. Louis, USA) were mixed with $7 \mathrm{ml}$ of double-distilled water and crosslinked for $2-3 \mathrm{~min}$ at $25^{\circ} \mathrm{C}$ using $2 \mathrm{ml}$ of $1 \%$ 1-ethyl-3-(3-dimethylaminopropyl)-carbodiimide (EDAC), the $\mathrm{pH}$ of the solution being 5-6. The solution was frozen at $-20^{\circ} \mathrm{C}$ for $1 \mathrm{~h}$, frozen at $-70^{\circ} \mathrm{C}$ for $1 \mathrm{~h}$, then lyophilized for $72 \mathrm{~h}$. The dried scaffold was re-crosslinked for $24 \mathrm{~h}$ at room temperature, using $10 \mathrm{ml}$ of $0.2 \%$ EDAC, then lyophilized for $72 \mathrm{~h}$. A tri-copolymer scaffold disc about $5 \mathrm{~cm}$ in diameter and $1 \mathrm{~cm}$ thick was produced which was cut into small scaffold cylinders $5 \mathrm{~mm}$ in diameter and $5 \mathrm{~mm}$ thick.

\subsubsection{Preparation of scaffold for SEM}

The scaffold was dehydrated by treatment with a series of graded ethanol solutions $(50 \%$ for $12 \mathrm{~h}$, then $75 \%, 85 \%$, and $95 \%$, each for $2 \mathrm{~h}$ ), then placed overnight in a vacuum oven at $50^{\circ} \mathrm{C}$ before coating with gold for scanning electron microscope (SEM) examination.

\subsection{Isolation of chondrocytes}

Full thickness articular cartilage was harvested aseptically from adult porcine knee joints within $12 \mathrm{~h}$ after slaughter and chondrocytes isolated by incubating the cartilage specimens for $12-16 \mathrm{~h}$ at $37^{\circ} \mathrm{C}$ in DMEM medium (Hyclone Co., Logan, UT, USA) containing $0.2 \%$ collagenase (Sigma Co., St. Louis, USA). The isolated chondrocytes were re-suspended in phosphatebuffered saline ( $\mathrm{pH}$ 7.4), washed, and counted using a hemocytometer. Chondrocyte viability was determined using Trypan blue dye exclusion.

\subsection{Seeding and culturing of chondrocytes}

Lyophilized scaffold cylinders $(5 \mathrm{~mm}$ long, $5 \mathrm{~mm}$ diameter) were sterilized with $75 \%$ ethanol. Chondrocytes were expanded in DMEM containing 10\% fetal bovine serum (Biological Industries Ltd, Kibbutz Beit Haemek, Israel), 1\% penicillin/gentamicin (Sigma Co., St. Louis, USA), and $50 \mu \mathrm{g} / \mathrm{ml}$ of L-ascorbic acid (Sigma Co., St. Louis, USA). At confluence, the cells were trypsinized and re-suspended at a concentration of $5.3 \times 10^{7}$ cells $/ \mathrm{ml}$ DMEM, then about $100 \mu \mathrm{l}$ of cell suspension was injected into each scaffold $\left(5.3 \times 10^{6}\right.$ cells per scaffold). The cell-containing scaffolds were then cultured in Petri dishes or in a spinner flask bioreactor for 2, 3, 4, and 5 weeks. The medium in the spinner flask was changed every week and the monolayer cultures subcultured after a week. The spinner flask was stirred at $70 \mathrm{rpm}$ for $10 \mathrm{~h}$ to distribute the cells more evenly, then at $50 \mathrm{rpm}$ during the rest of the culture period. Quadruplicate samples were removed at each time-point and fixed with formaldehyde, then stained with $\mathrm{H} \& \mathrm{E}$ stain for histological examination. Alcian Blue was used to stain glycosaminoglycan and antibodies against human S-100 protein (Novocastra Laboratories Ltd, Newcastle upon Tyne, UK) or type II collagen (Santa Cruz Biotech. Inc., CA, USA) to check the chondrocyte phenotype. 


\section{Results and discussion}

\subsection{Scanning electron microscopy and light microscopy}

An SEM photograph of the gelatin tri-copolymer is shown in Fig. 1. The tri-copolymer scaffold had a uniform pore size of $180 \mu \mathrm{m}$ and a porosity of $75 \%$. This highly porous structure should allow cell penetration, growth, and proliferation; in fact, the pore size increased when the scaffold was rehydrated in culture medium (Fig. 2).

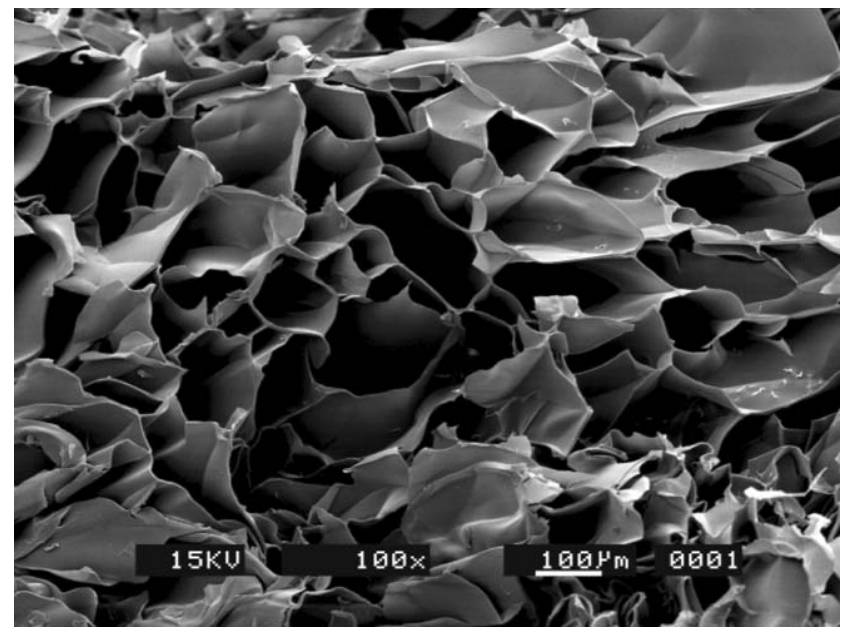

Fig. 1. Scanning electron microscopic examination of the gelatin/ chondroitin-6-surfate/hyaluronan tri-copolymer scaffold showing a porous structure with a uniform pore size of about $180 \mu \mathrm{m}$ and a porosity of $75 \%$.

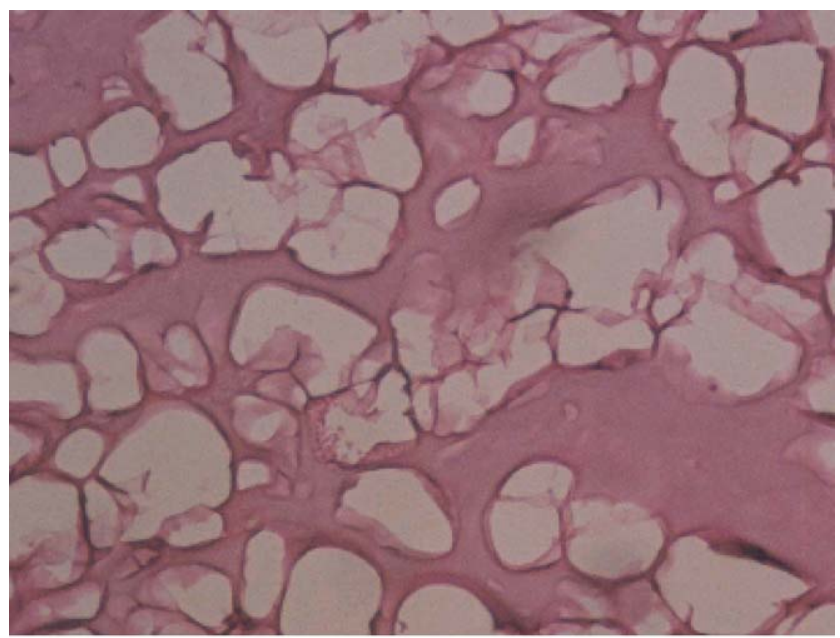

$150 \mu \mathrm{m}$

Fig. 2. Gelatin/chondroitin-6-sulfate/hyaluronan tri-copolymer scaffold stained with $\mathrm{H} \& \mathrm{E}$ and examined by light microscopy. The scaffold stains reddish and shows uniform porosity.

\subsection{Culture in Petri dishes and spinner flasks}

\subsubsection{Cell distribution}

When the scaffolds were seeded with chondrocytes and cultured in Petri dishes or spinner flasks for 3 weeks, the distribution of the chondrocytes in the scaffold was more homogenous in the spinner flasks than in the Petri dishes, the cells in the Petri dishes being distributed as aggregates in the superficial areas of the scaffold (Fig. 3(a)). A homogenous distribution of attached cells is necessary for the development of engineered tissue and the spinner flask is the preferred system for seeding chondrocytes on a poly-glycolic acid (PGA) mesh [12]. Our results showed that the spinner flask was also effective in seeding chondrocytes into a gelatin-chondroitin sulfate-hyaluronan tri-copolymer scaffold with a uniform spatial distribution (Fig. 3(b)).

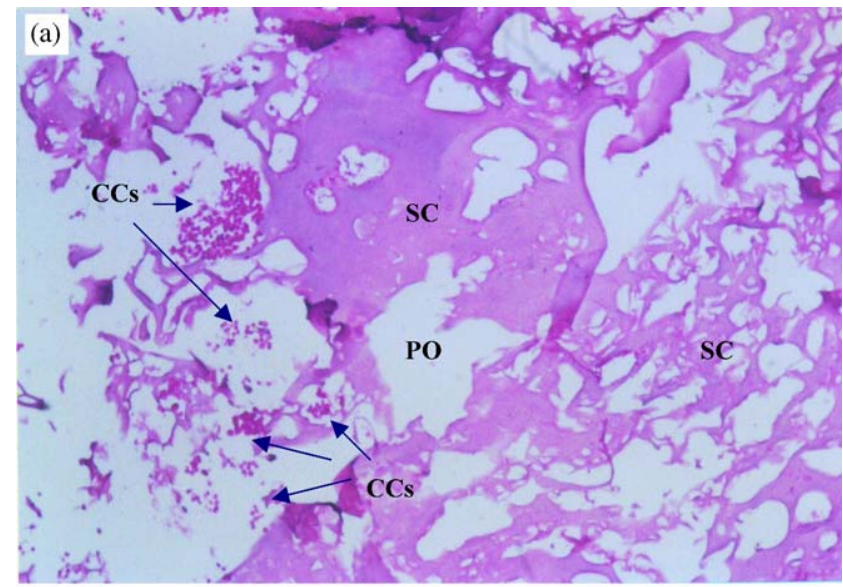

$\underline{80 \mu \mathrm{m}}$

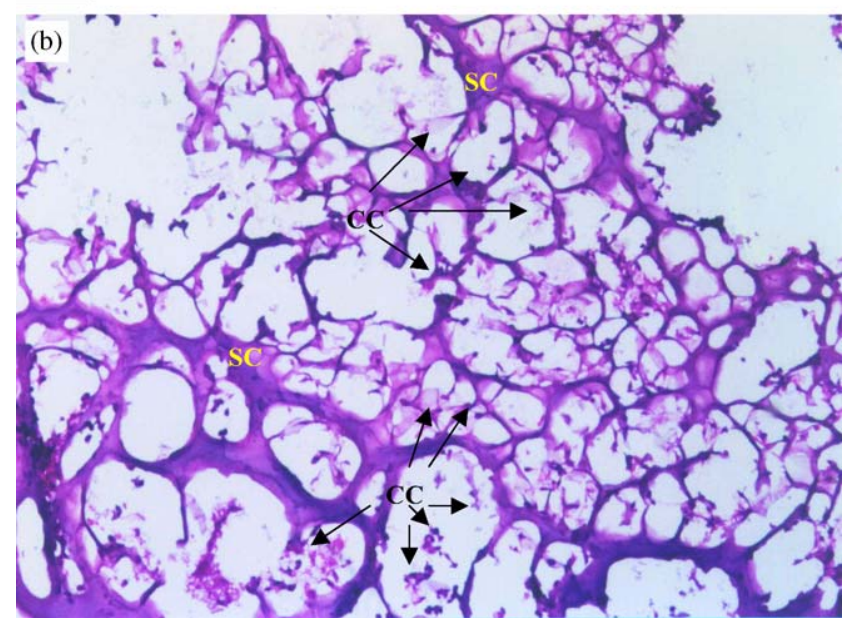

Fig. 3. Chondrocyte distribution in scaffold after 3 weeks of culture (a) Petri dish culture and (b) spinner flask culture. Petri-dish-grown cells were aggregated on the surface of the scaffold, while spinner flask cultures showed a homogenous distribution of chondrocytes in the scaffold. SC: scaffold; CCs: clustered chondrocytes; CC: chondrocytes; PO: pore $(40 \times)$. 


\subsubsection{Secretion of ECM}

Because of the better distribution obtained using spinner flasks, subsequent studies were performed using this system. Secretion of ECM by chondrocytes was not seen in the first 3 weeks, but occurred after 4 weeks of culture (Fig. 4). Lacuna formation was seen in the matrix surrounding the chondrocytes, the morphology of these lacunas being similar to that of natural cartilage. On histological examination of the specimens in 5 weeks' cultivation, this newly formed matrix was stained light blue with Alcian blue, showing glycosaminoglycan secretion (Fig. 5). The new matrix was less

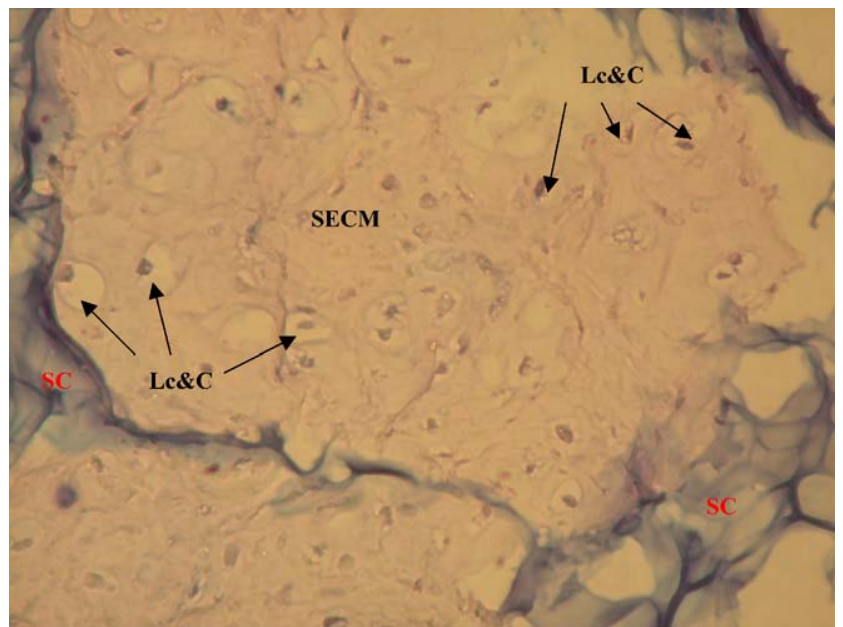

$25 \mu \mathrm{m}$

Fig. 4. After 4 weeks' cultivation in spinner flask, newly secreted matrix with lacuna formation around chondrocytes as shown by Alcian Blue staining. The morphology of the lacunas is similar to those seen in natural cartilage. Lc\&C: lacuna and chondrocytes; SECM: secreted extracellular matrix; SC: scaffold $(200 \times)$.

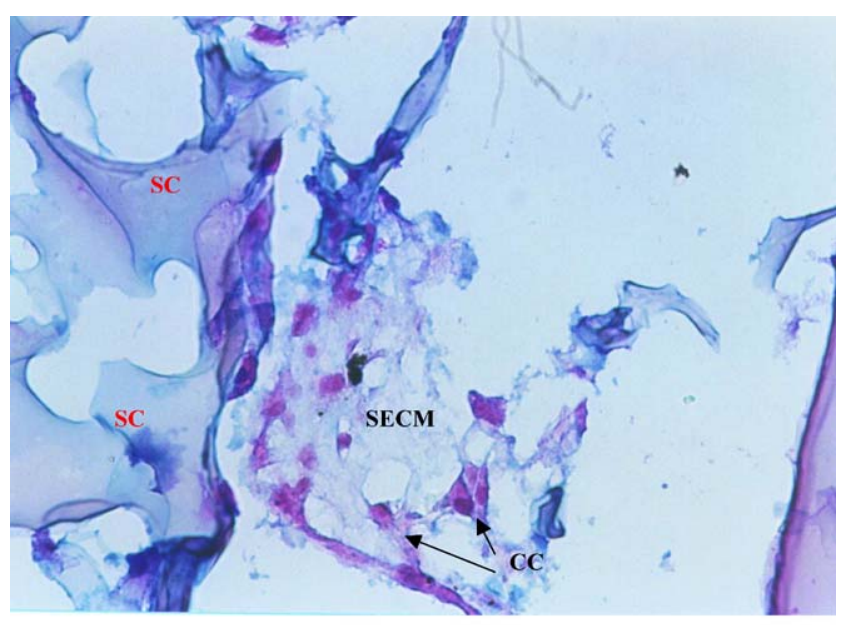
$25 \mu \mathrm{m}$

Fig. 5. After 5 weeks' cultivation in spinner flask, Alcian Blue staining showing that the peri-cellular matrix around chondrocytes stains light blue, while the tri-copolymer scaffold stains darker, indicating that the scaffold contains more glycosaminoglycan. SC: Scaffold; SECM: secreted extracellular matrix, CC: chondrocytes $(200 \times)$. strongly stained than the scaffold with $\mathrm{H} \& \mathrm{E}$ or Alcian blue, showing that it contained less protein (gelatin) and glycosaminoglycan (chondroitin-6-sulfate) than scaffold.

This newly formed matrix contained regenerated collagen fibers with an organized structure with anisotropic properties, as shown by polarized microscopy (Fig. 6), whereas no polarization was seen in the gelatincontaining scaffold, as gelatin has an amorphous crystal structure with isotropic properties. Polarized microscope is therefore a good method for differentiating the newly formed matrix from the matrix-mimicking scaffold.

\subsubsection{Retention of chondrocyte phenotype}

Immunohistochemical staining for S-100 protein and type II collagen showed that chondrocytes cultured in spinner flasks for 5 weeks retained their phenotype in the tri-copolymer scaffold. As shown in Fig. 7, the cells retained their round shape and stained positive for S-100 protein. The round shape of chondrocytes is an indicator of phenotype retention and is essential for matrix formation [13]. S-100 protein is only found in cells of ectodermal origin and chondrocytes are the only cell of mesenchymal origin to express S-100 protein; S-100 expression is lost when chondrocytes lose their phenotype [14]. As shown in Fig. 8, the chondrocytes were also stained with anti-type II collagen antibody. Type II collagen, which is essential for articular cartilage, is produced by chondrocytes; these results therefore show that the chondrocytes in the scaffold were functionally active.

Sechriest et al. [10] found that chondroitin sulfate in the scaffold promotes the secretion of proteoglycan and type II collagen, but inhibits mitosis. Hyaluronan is present at high amounts in the ECM during embryonic

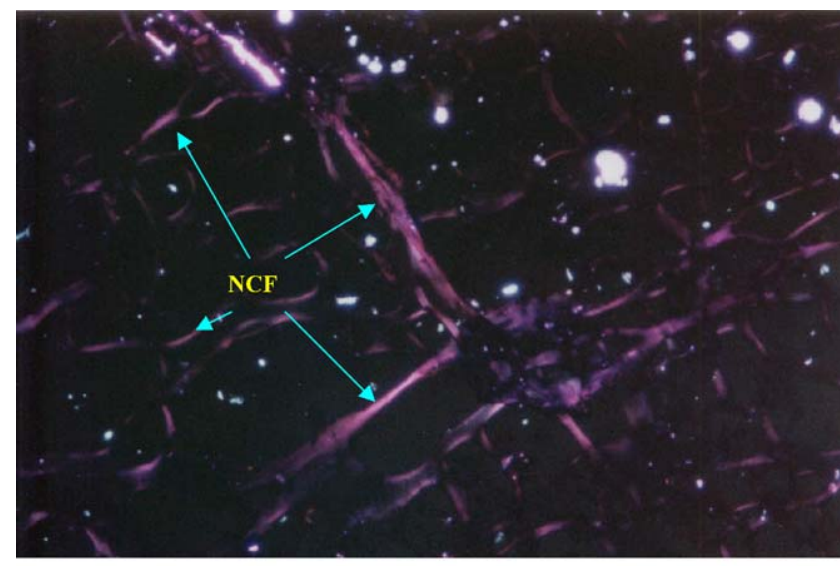

\section{$\underline{40 \mu \mathrm{m}}$}

Fig. 6. Polarized microscopic examination after 5 weeks' cultivation in spinner flask, showing that the newly formed matrix contains organized collagen fibers with anisotropic properties not seen in the region of the scaffold. NCF: newly formed collagen fiber. 


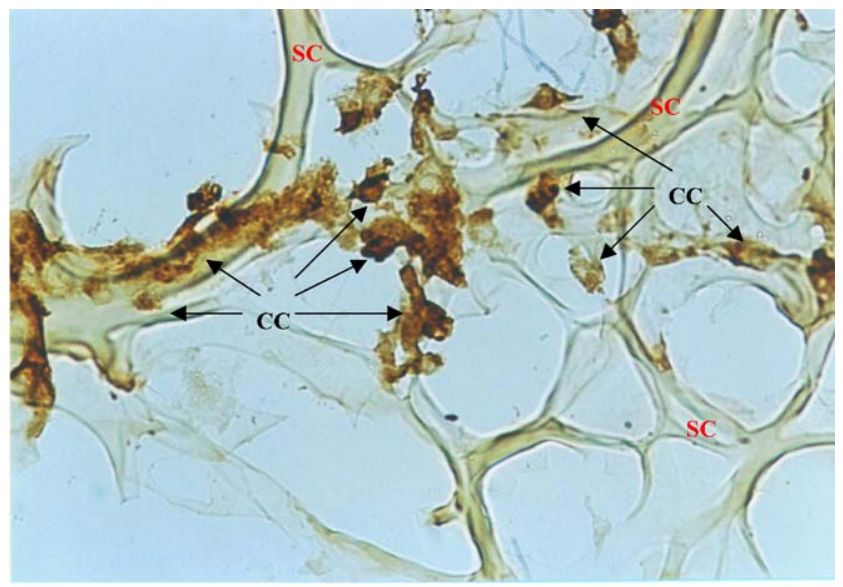

$25 \mu \mathrm{m}$

Fig. 7. Phenotype retention by the chondrocytes after 5 weeks' cultivation in spinner flask. Chondrocytes retained their round shape and were stained with anti-S-100 protein antibody $(200 \times)$.

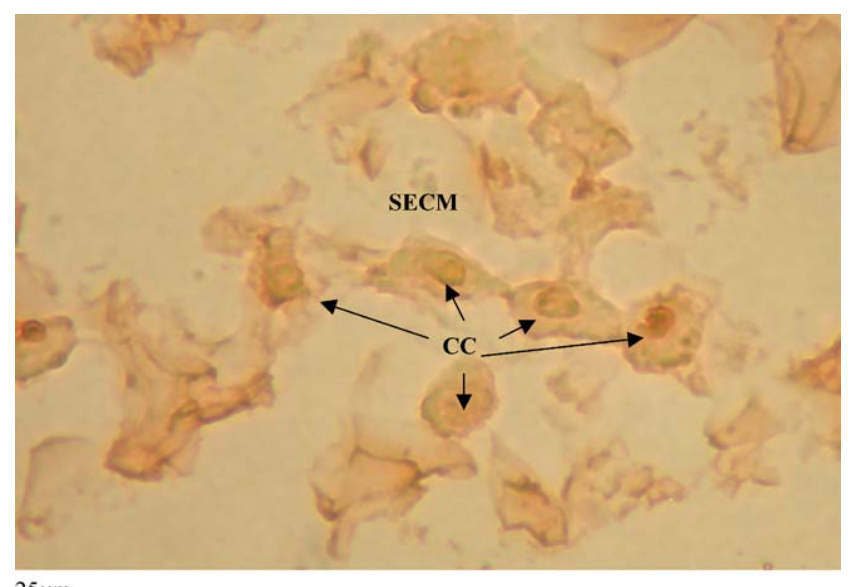

$25 \mu \mathrm{m}$

Fig. 8. The newly formed matrix produces type II collagen after 5 weeks' cultivation in spinner flask. The matrix was stained with antitype II collagen antibody. SECM: secreted extracellular matrix, CC: chondrocytes $(200 \times)$.

cartilage development and also facilitates the integration of engineered cartilage [5]; small oligomers of hyaluronan can remove aggrecan from the cartilage matrix, thereby providing space for newly synthesized matrix components from the neotissue to bridge into the host tissue [5]. We hypothesized that a tri-copolymer formed from gelatin, chondroitin, and hyaluronan might mimic cartilage matrix and provide information for cell attachment to meet the requirement for dynamic reciprocity for cartilage tissue engineering. In this study, the distribution of chondrocytes in the scaffold was better in the spinner flask bioreactor than in Petri dishes. Our results show that chondrocytes survived well in this tri-copolymer scaffold and secreted newly synthesized matrix, which was less stained with Alcian Blue than the chondroitin-6-sulfate-containing scaffold. However, the amount of synthesized matrix is still not enough to form cartilage. Since a study in animal model has shown that more than 6 weeks period is required to repair cartilage [15], a further study using a longer culture period is required. The less than satisfactory amount of matrix production may also be due to inhibition of cell mitosis by chondroitin, so experiments using scaffolds formed without chondroitin are planned.

\section{Conclusion}

As tissue engineering is now showing promise as a possible method for cartilage repair, seeking a suitable scaffold has become more and more important. Traditionally, cartilage tissue engineering studies have used PGA, poly-L-lactic acid (PLA), or a copolymer of PGA and PLA (PLGA) to make scaffold [16]. However, these materials have certain shortcomings in that they are hydrophobic and need pre-wetting before cell seeding [17], are non-biological and lack informational structure, such as the Arg-Gly-Asp sequence for cell attachment, and their degradation products, e.g., glycolic acid and lactic acid, are acidic and lower the $\mathrm{pH}$ around tissue after in vivo implantation, which may cause severe inflammation. This inflammatory response appears to be a direct consequence of the chemical composition of the polymer degradation products, for which there is currently no prophylactic measure [18]. Gelatin, a denatured collagen, is a biological material, is thought to provide information for cell attachment, and has good biocompatibility. The reason for choosing the gelatin/chondroitin sulfate/hyaluronan tri-copolymer in this study was to mimic the natural cartilage matrix and to try to meet the requirement for dynamic reciprocity for cartilage tissue engineering. There have been no previous reports of the use of this tri-copolymer for cartilage tissue engineering. Collagen and glycosaminoglycan (GAG) are principal components of the normal ECM. A collagen-GAG copolymer has been used as scaffold for an artificial skin graft [19], and should be more biocompatible and less immunogenic than traditional polymers, such as PGA, be degradable, and produce non-toxic metabolites. Copolymerization of GAG with collagen yields sponges that are more degradation-resistant and have higher elastic moduli and higher fracture energy than collagen alone [20]. These experiences with artificial skin lead us to believe that a gelatin/chondroitin sulfate/hyaluronan tri-copolymer might serve as a better scaffold for cartilage tissue engineering than synthetic polymers. The results of the present study support this hypothesis. When cultured in spinner flasks, chondrocytes are evenly distributed in the scaffold, secrete new ECM, retain their phenotype, and secrete type II collagen. The gelatin/chondroitin sulfate/ hyaluronan tri-copolymer therefore deserves to be tested 
as a candidate for cartilage tissue engineering. More ECM secretion with lacuna formation and more GAG production might be expected after longer periods of culture.

\section{Acknowledgements}

The authors were supported by Grant B900009 from the Far Eastern Memorial Hospital and Far Eastern Medical Foundation and NSC-91-NU-7-002-001.

We also thank Dr. Tom Barkas for revising the English.

\section{References}

[1] Gillogly SD, Voight M, Blackburn T. Treatment of articular cartilage defects of the knee with autologous chondrocyte implantation. J Orthop Sports Phys Ther 1998;28(4):241-51.

[2] Gilbert JE. Current treatment options for the restoration of articular cartilage. Am J Knee Surg 1998;11:42-6.

[3] Mitchell N, Shepard N. The resurfacing of adult rabbit articular cartilage by multiple perforations through the subchondral bone. J Bone Jt Surg 1976;58A:230-3.

[4] Hunziker EB. Articular cartilage repair: basic science and clinical progress. A review of the urrent status and prospects. Osteoarthritis Cartilage 2002;10:432-63.

[5] Solchaga LA, Goldberg VM, Caplan AI. Cartilage regeneration using principles of tissue engineering. Clin Orthop 2001; 391S:S161.

[6] Athanasiou KA, Shah AR, Hernandez RJ, LeBaron RG. Basic science of articular cartilage repair. Clin Sports Med 2001;20(2):223-47.

[7] Solchaga LA, Yoo JU, Lundberg M, Dennis JE, Huibregtse BA, Goldberg VM, Caplan AI. Hyaluronan-based polymers in the treatment of osteochondral defects. J Orthop Res 2000;18(5): $773-80$.
[8] Ruoslahti E, Pierschbacher MD. Arg-Gly-Asp: a versatile cell recognition signal. Cell 1986;44:517-8.

[9] Scully SP, Lee JW, Ghert PMA, Qi W. The role of the extracellular matrix in articular chondrocyte regulation. Clin Orthop 2001;391S:S72.

[10] Sechriest VF, Miao YJ, Niyibizi C, Westerhausen-Larson A, Matthew HW, Evans CH, Fu FH, Suh JK. GAG-augmented polysaccharide hydrogel: a novel biocompatible and biodegradable material to support chondrogenesis. J Biomed Mater Res 2000;49(4):534-41.

[11] Maroudas A. Physiochemical properties of articular cartilage in adult articular cartilage, 2nd ed. Kent, UK: Pitman Medical Publishing Co.; 1979. p. 215-290.

[12] Vunjak-Novakovic G, Obradovic B, Bursac P, Martin I, Langer R, Freed LE. Dynamic seeding of polymer scaffolds for cartilage tissue engineering. Biotechnol Prog 1998;14:193-202.

[13] von der Mark K, Gauss V, von der Mark H, Muller P. Relationship between cell shape and type of collagen synthesised as chondrocytes lose their cartilage phenotype in culture. Nature 1977;267(5611):531-2.

[14] Wolff DA, Stevenson S, Goldburg VM. S-100 protein immunostaining identifies cells expressing a chondrocytic phenotype during articular cartilage repair. J Orthop Res 1992;10:49-57.

[15] Hunziker EB, Rosenberg LC. Repair of partial thickness defects in articular cartilage: cell recruitment from the synovial membrane. J Bone Jt Surg 1996;78A(5):721-33.

[16] Lu L, Zhu X, Valenzuela RG, Currier BL, Yazemski MJ. Biodegradable polymer scaffolds for cartilage tissue engineering. Clin Orthop 2001;391S:S251

[17] Freed LE, Vunjak-Novakovico G. Tissue culture bioreactors. In: Lanza RP, Langer R, Vancanti JP, editors. Principles of tissue engineering, 2nd ed. San Diego: Academic Press; 2000. p. 143-56.

[18] Bostman OM, Pihlajamaki HK. Adverse tissue reactions to bioabsorbable fixation devices. Clin Orthop 2000;371:216-27.

[19] Boyce ST, Christianson DJ, Hansbrough JF. Structure of a collagen-GAG dermal skin substitute optimized for cultured human epidermal keratinocytes. J Biomed Mater Res 1988;22:939-57.

[20] Cahn F. Modification of natural polymers: collagen-glycosaminoglycan copolymers. In: Atala A, Lanza RP, editors. Methods in tissue engineering. San Diego: Academic Press; 2002. p. 515-23. 